\title{
PRP18, a Protein Required for the Second Reaction in Pre-mRNA Splicing
}

\author{
USHA VIJAYRAGHAVAN AND JOHN ABELSON* \\ Division of Biology 147-75, California Institute of Technology, Pasadena, California 91125
}

Received 25 July 1989/Accepted 12 October 1989

\begin{abstract}
We have investigated the role of a novel temperature-sensitive splicing mutation, prp18. We had previously demonstrated that an accumulation of the lariat intermediate of splicing occurred at the restrictive temperature in vivo. We have now used the yeast in vitro splicing system to show that extracts from this mutant strain are heat labile for the second reaction of splicing. The heat inactivation of prp 18 extracts results from loss of activity of an exchangeable component. Inactivated prp18 extracts are complemented by heat-inactivated extracts from other mutants or by fractions from wild-type extracts. In heat-inactivated prp18 extracts, $40 \mathrm{~S}$ splicing complexes containing lariat intermediate and exon 1 can assemble. The intermediates in this $40 \mathrm{~S}$ complex can be chased to products by complementing extracts in the presence of ATP. Both complementation of extracts and chasing of the isolated prp18 spliceosomes takes place with micrococcal nuclease-treated extracts. Furthermore, the complementation profile with fractions of wild-type extracts indicates that the splicing defect results from a mutation in a previously designated factor required for the second step of splicing. The isolation of this mutant as temperature-sensitive lethal has also facilitated cloning of the wild-type allele by complementation.
\end{abstract}

The recognition and splice site selection in nuclear premRNA splicing is dictated by three short stretches of sequence elements in the intron (34). The specificity of splice junction selection and the juxtaposition of the exons is achieved by the recognition of these cis-acting conserved elements in the intron by cellular factors that include proteins and small nuclear ribonucleoprotein particles (snRNPs) (reviewed in references 28 and 35). The pre-mRNA is spliced in a large multicomponent structure termed the spliceosome $(2,5,10,11,30)$. Splicing reactions that take place in the spliceosome occur via a two-step mechanism. First, the pre-mRNA is cleaved at the $5^{\prime}$ splice junction, and this results in the formation of the reaction intermediates: exon 1 and a lariat intron-exon 2 structure in which the first base of the intron is covalently linked to a specific adenosine near the $3^{\prime}$ splice site. The second reaction involves cleavage at the $3^{\prime}$ splice site and the ligation of the two exons to produce the mature mRNA and the lariat intron (for reviews, see references 12 and 29 ).

The complexity of the splicing reaction and the large particle in which the reactions take place suggests the need for multiple functions. At least five small nuclear RNAs (snRNAs) (U1, U2, U4, U5, and U6) are involved in the splicing of both yeast and higher eucaryotic pre-mRNA (13, 38). These snRNAs, functioning in the form of snRNPs, are associated with active splicing complexes. The spliceosome assembly intermediates resolved on nondenaturing gels vary in the order of appearance and their snRNP composition. U1 binds to the pre-mRNA first, and this is followed by the binding of $U 2$, U4, U5, and U6 snRNPs $(3,8,16,31,32)$. The requirement in splicing for protein factors, apart from snRNPs, has been demonstrated through biochemical fractionation of splicing extracts $(7,18,19)$.

Analysis of splicing factors in Saccharomyces cerevisiae can readily use a combination of biochemical, classical genetic, and molecular approaches. Such an approach has

\footnotetext{
* Corresponding author
}

taken advantage of a set of temperature-sensitive mutations (rna2 to rnall) that affect pre-mRNA splicing in vivo for the analysis of splicing factors (for reviews, see references 40 and 42). Henceforth, all the rna mutants will be called pre-RNA processing ( $p r p$ ) mutants. In vitro heat inactivation of splicing extracts from many of these prp complementation groups has implied a direct role for these gene products in pre-mRNA splicing. Consistent with the in vivo observation of pre-mRNA accumulation at nonpermissive temperatures in all of the prp2 to prp11 strains, inactivation of both steps of splicing was observed upon heating of splicing extracts from most of the mutants (26). In addition, most of the $P R P$ gene products are required for spliceosome formation itself (23). The exception is the $P R P 2$ gene product, which acts after spliceosome assembly and is required for the first splicing reaction. Studies on the role of PRP2 in in vitro splicing systems have suggested the need for several extrinsic and intrinsic factors for both splicing reactions (23). In addition to the in vitro characterization of the prp2 to prpll mutants, many of these genes have been cloned and their molecular characterization has been initiated $(15,20-22,37$; for a review, see reference 40). Antibodies directed against PRP4, PRP8, and PRP11 proteins inhibit the in vitro splicing reaction, reaffirming their essential role in splicing $(1,6,15)$. The PRP8 protein has been demonstrated to associate with U5 snRNA (25). The association of the PRP4 protein with the U4/U6 snRNAs has recently been shown (1). The PRP11 protein associates with spliceosomes and is also present in a preassembled $30 \mathrm{~S}$ complex.

We have recently isolated a new set of temperaturesensitive mutations affecting pre-mRNA processing (41). Isolation of these 11 new complementation groups affecting pre-mRNA splicing in vivo was done by a direct search for such mutants. Interestingly, four groups with novel phenotypes were obtained which specifically affected late stages of splicing: (i) cleavage at the $3^{\prime}$ splice site and exon ligation and (ii) degradation of the lariat intron. These mutants facilitate the development of assays for specific steps in 
splicing, and therefore the analysis and purification of specific splicing factors. The isolation of two complementation groups (prp17 and prp18) with the phenotype of lariat intermediate accumulation allows analysis of splicing components required for the second reaction of splicing. In these mutants, the lariat accumulation results from a specific defect affecting the second splicing reaction, as no premRNA accumulates at the nonpermissive temperature. Studies on the prp2 heat-inactivated spliceosomes have suggested the requirement for two sets of factors for the second splicing reaction (23). In mammalian systems also, at least two factors have been reported to be required at this stage of splicing $(18,19)$.

A combination of biochemical and molecular analyses has been initiated to study the role of PRP18 in splicing. We report that a mutation in one of the previously hypothesized $S$. cerevisiae splicing factors results in lariat intermediate accumulation. A specific requirement for ATP in the second step of splicing was also observed. We also report the cloning of the wild-type PRP18 locus.

\section{MATERIALS AND METHODS}

Strains and microbiological techniques. The wild-type $S$. cerevisiae strains used in this study were EJ101 and SS330. The prp5 strain was RL172, and the prp11 strain was SPJ11.4. The genotypes of these strains are as described by Lustig et al. (26) and Vijayraghavan et al. (41). The prp18-2 strains were obtained after outcrossing temperature-sensitive $(t s)$ strain 503 with wild-type strain SS330 and were 503-1a MATa ade2-101 his3 $\Delta 200$ ura3-52 lys2-801 and 503-6c MATa ade2-101 his $3 \Delta 200$ ura3-52 lys 2-801. The yeast strains were propagated by standard methods on media as described by Sherman et al. (36). Temperature-sensitive strains were maintained at $23^{\circ} \mathrm{C}$, and $37^{\circ} \mathrm{C}$ was used as the nonpermissive temperature. Sporulation, omission, and preparation of rich media were done as described by Sherman et al. (36). Yeast transformations were all done by the lithium acetate procedure of Ito et al. (14). Transformants were selected on omission media at $23^{\circ} \mathrm{C}$, and colonies were then replica plated to prewarmed rich plates (YPD) at $37^{\circ} \mathrm{C}$ to test for temperature resistance. The YCp50-based Sau3A library of $S$. cerevisiae genomic DNA was obtained from $M$. Rose and P. Novick. The yeast shuttle vectors pPHY18 (CEN ARS URA3 Amp') and pSEY18 ( $2 \mu \mathrm{m} A R S$ URA3 Amp') used in subcloning experiments were kindly provided by $\mathrm{P}$. Herman and S. Emr. Selection for the loss of yeast CEN URA3 plasmids was done on 5-fluoro-orotic acid plates prepared as described by Boeke et al. (4). The Escherichia coli strain used for cloning and DNA amplification was MC1061 [F $\mathrm{F}^{-}$ araD139 $\Delta($ araABIOC-leu $) 7679 \Delta$ (lac) X74 galU galK rpsL hsr hsm $^{+}$].

Nucleic acid manipulations. Restriction mapping and standard cloning techniques were done essentially as described by Maniatis et al. (27). Subcloning of restriction fragments from the plasmids was done after isolation of the desired restriction fragment and of the suitably digested vectors (pPHY18, pSEY18, or YIP5) from low-melting-point agarose gels. The plasmids from $E$. coli cells were analyzed after alkali lysis preparation of DNA. Probes, when used, were restriction fragments obtained from low-melting-point agarose gels and were generated with the random primer-labeling procedure of Feinberg and Vogelstein (9). Small-scale preparations of yeast genomic DNA were done as described by Sherman et al. (36). Southern blots were performed as suggested by Dupont, NEN Research Products. Hybridiza- tions were conducted in conditions similar to those described by Cheng and Abelson (8).

Preparation of total RNA from yeast was done as described by Vijayraghavan et al. (41). The RNA was separated on formaldehyde-agarose gels before being blotted onto a nylon membrane. Primer extension on $20 \mu \mathrm{g}$ of total RNA, with an RP51 oligonucleotide hybridizing 70 nucleotides downstream of the $5^{\prime}$ splice site as a primer, was performed with reverse transcriptase as described by Vijayraghavan et al. (41).

Preparation of splicing extracts. Extracts were isolated from mid- to late-logarithmic-phase cells grown in YPD at $23^{\circ} \mathrm{C}$ by the spheroplast procedure of Lin et al. (24). The protein concentration of the extracts was approximately 20 $\mathrm{mg} / \mathrm{ml}$. Fractions 40P3 and 40W from extracts of wild-type EJ101 cells were provided by S.-C. Cheng.

In vitro splicing reactions, heat inactivation, and complementation. SP6 actin transcripts were generated by using SP6 polymerase with UTP at $100 \mu \mathrm{M}$ and $\left[^{32} \mathrm{P}\right] \mathrm{UTP}$ at 2 $\mathrm{mCi} / \mathrm{ml}$. Heat inactivation of splicing extracts involved the use of $7 \mu \mathrm{l}$ of extract to which was added $2.2 \mu \mathrm{l}$ of an inactivation cocktail such that inactivation conditions were $2.4 \mathrm{mM} \mathrm{MgCl}-1 \mathrm{mM}$ dithiothreitol-12 $\mathrm{U}$ of $\mathrm{RNasin}$. Inactivation was done at 30 to $32^{\circ} \mathrm{C}$ for various periods, and time point samples were taken and kept on ice until completion of the last time point, at which time splicing was initiated. Splicing cocktail was added to obtain a final concentration of $2.2 \mathrm{mM}$ ATP-55 mM KPO $-2.7 \%$ polyethylene glycol, with 5,000 to $10,000 \mathrm{cpm}(\sim 3.3 \mathrm{fmol})$ of labeled actin pre-mRNA. Splicing was carried out at $15^{\circ} \mathrm{C}$ for 30 to $60 \mathrm{~min}$, and the reaction mixture was phenol extracted and ethanol precipitated. The precipitated RNA was analyzed on denaturing polyacrylamide gels as described by Lin et al. (24). Complementation in vitro was tested by mixing approximately equal amounts of individually inactivated extracts before initiation of splicing. Fractions of wild-type extract were diluted in inactivation cocktail and were then added to inactivated prp18 extracts.

Glycerol gradient sedimentation of splicing reactions. Splicing reaction mixtures $(50 \mu \mathrm{l})$ were chilled on ice and diluted with cold gradient buffer $\left(0.1 \mathrm{M} \mathrm{KCl}-2 \mathrm{mM} \mathrm{MgCl}_{2}-20 \mathrm{mM}\right.$ HEPES [ $N$-2-hydroxyethylpiperazine- $N^{\prime}$-2-ethanesulfonic acid], $\mathrm{pH}$ 7.4) such that the density of the sample was lower than that of the top of the gradient. The diluted sample was layered carefully on a 15 to $27 \%$ glycerol gradient made in 0.5 - to 2 -inch (1.3- to 5-cm) polyallomer tubes. The sedimentation was done at $50,000 \mathrm{rpm}$ for about $100 \mathrm{~min}$ at 2 to $4^{\circ} \mathrm{C}$ in an SW55 rotor. Fractions were collected on ice, and Cerenkov counts of the fractions were determined. Fractions of interest were frozen in liquid $\mathrm{N}_{2}$ and stored at $-70^{\circ} \mathrm{C}$. A small sample from each fraction across the $40 \mathrm{~S}$ region of the gradient was phenol extracted and ethanol precipitated. The samples were analyzed on nondenaturing gels to confirm that the $40 \mathrm{~S}$ region contained the expected labeled RNA molecules.

Chasing reaction of gradient-isolated spliceosomes. Conversion of the lariat intermediate and exon 1 to products (lariat intron and mRNA) was termed a chasing reaction. Typically, $20-\mu l$ reaction mixtures with $14 \mu$ l of the appropriate gradient fraction, $1 \mu$ l of $10 \times$ chasing buffer $\left(200 \mathrm{mM} \mathrm{KPO}_{4}, \mathrm{pH} \mathrm{7,11}\right.$ $\mathrm{mM} \mathrm{MgCl} 2,20 \mathrm{mM}$ ATP, 14\% polyethylene glycol 8000) and $2 \mu \mathrm{l}$ of complementing extract were incubated at $15^{\circ} \mathrm{C}$ for 60 min. Fractions of wild-type extract when used for complementation were added at one-half the volume of other complementing extracts. The reaction products were recov- 


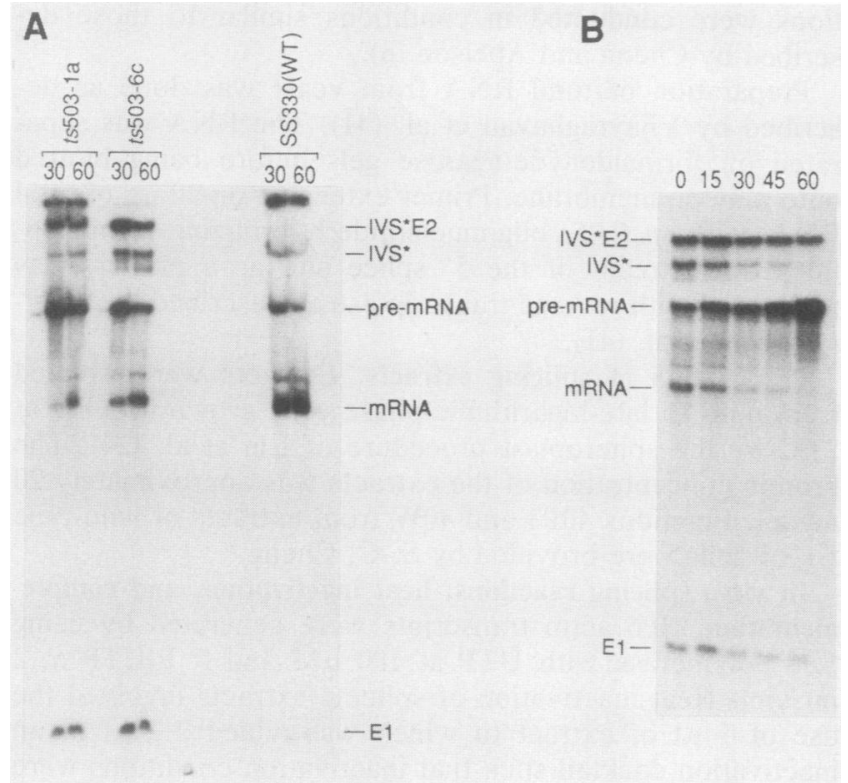

FIG. 1. (A) Splicing activity in prp18 and wild-type extracts. Splicing assays were conducted at $15^{\circ} \mathrm{C}$ for 30 or $60 \mathrm{~min}$ as indicated. Extracts from two prp18 strains ( $t s$ 503-1a and $t s$ 503-6c) and from the wild-type strain SS330 were assayed. These extracts were used without any heat inactivation, with splicing conditions as described in Materials and Methods. Migration positions of the lariat intermediate (IVS*E2), the lariat intron (IVS*), spliced exons (mRNA), and exon 1 (E1) are indicated. (B) Heat inactivation of splicing extracts from prp18. $t s$ 503-6c extracts were inactivated under the conditions described in Materials and Methods for increasing periods of time (0 to $60 \mathrm{~min}$ ) at $32^{\circ} \mathrm{C}$. After inactivation, splicing was initiated with the addition of labeled actin pre-mRNA in a cocktail, and splicing was conducted at $15^{\circ} \mathrm{C}$ for $60 \mathrm{~min}$. Reaction products were fractionated on denaturing acrylamide gels. RNA species labeled IVS*E2, IVS*, pre-mRNA, mRNA, and E1 are explained above.

ered by phenol-chloroform extraction and were separated on polyacrylamide gels.

\section{RESULTS}

Heat inactivation of splicing extracts from prp18. The isolation of a temperature-sensitive mutation affecting the second step of splicing in vivo provides a route for the study of factors required in the second stage of the splicing reaction. To do this, we have examined the effects of the prp18 mutation on in vitro mRNA splicing. Whole-cell splicing extracts capable of in vitro splicing were prepared from strains bearing the prp18 mutation. Extracts from cells grown at the permissive temperature were assayed for splicing activity at permissive temperature in vitro $\left(15^{\circ} \mathrm{C}\right)$. The splicing activity was comparable with that of wild-type extracts. The first step of splicing seemed to be unaffected; however, the extent of the second reaction of splicing in different prp18 extracts was variable (Fig. 1A; compare activities in prp 18-2 strains $t s 503-1 \mathrm{a}$ and $t s 503-6 \mathrm{c})$. Hence, the efficiency of cleavage at the $3^{\prime}$ splice site and the ligation of the exons appeared to be affected in whole-cell extracts prepared from this mutant strain.

To investigate the function of the $P R P 18$ gene product, heat inactivation of extracts from the prp18-2 strain ( $t s$ 503-6c) was carried out. Inactivation of extracts was done by heating them for 15 to $60 \mathrm{~min}$ at 30 to $32^{\circ} \mathrm{C}$ under the conditions described in Materials and Methods. The heat- inactivated extracts were assayed at the permissive temperature $\left(15^{\circ} \mathrm{C}\right.$ for $\left.60 \mathrm{~min}\right)$. In extracts from prp 18 , lariat intermediate and exon 1 accumulated and there was a substantial decrease in the levels of the mRNA and the lariat intron after 60 min of heat inactivation (Fig. 1B). The lack of an increase in the lariat intermediate and exon 1 levels with increasing preincubation time is most likely due to a nonspecific decrease in total splicing activity upon prolonged preincubation. Some wild-type extracts also exhibited a decrease in total splicing activity upon preincubation for a similar period (26; data not shown). It is possible that PRP18 has a secondary effect on early events of splicing, and this may account for the decrease in splicing efficiency with preincubation at $30^{\circ} \mathrm{C}$. However, this is unlikely to be the primary function of this splicing factor, since no detectable effect on early events in splicing, i.e., accumulation of pre-mRNA, was discernible in vivo (41).

In vitro complementation of inactivated extracts. Splicing assays with extracts prepared from prp18 strains accumulated lariat intermediate and exon 1 . One possible reason for this effect is denaturation or inactivation of a specific splicing component as a result of temperature sensitivity of the protein or RNA. To substantiate this theory, inactivated prp18 extracts were used to complement inactivated extracts from other temperature-sensitive strains. Complete inactivation of extracts from prp5 or prpll strains was obtained as previously described (26). Mixing of inactivated prpl8 extract with inactivated prp5 or prp11 extract resulted in some restoration of splicing activity (Fig. 2, lanes 1 and 2). A slightly higher level of complementation was achieved with inactivated prp 11 extract than with inactivated prp 5 extract. However, it is difficult to make a direct comparison because of the different splicing activities of prp 5 and prpll extracts themselves. A combination of $t s$ 503-6c extract with inactivated extracts from cells bearing the same prp 18 mutation in a different genetic background did not result in complementation (Fig. 2, lane 6). Thus, the loss of activity is due, at least in part, to inactivation of the PRP18 gene product; the complementation also indicates that some of the splicing factors can exchange under these assay conditions.

The defect in heat-inactivated prpl 18 extracts can also be complemented with fractions from a wild-type extract. We have used fraction I (40P3) and fractions II plus III (40W) obtained by ammonium sulfate precipitation (7). Complementation of the prp18 splicing defect is striking with 40P3 (fraction I) (Fig. 2, lane 3), while 40W does not seem to contain much complementing activity (Fig. 2, lane 4). We also tested whether inactivated prp 18 extract could be complemented by micrococcal nuclease-treated wild-type extracts. Micrococcal nuclease treatment of wild-type extracts (SS330) caused a complete loss of splicing activity (data not shown), as has been previously reported (7). Mixing the treated wild-type extract and the inactivated prpl8 extract restored splicing activity (Fig. 2, lane 5). Therefore, complementation of inactivated prp 18 extracts does not require the presence of intact $U 1, U 2, U 4$, or U6 snRNAs, although the complementing activity resides in an snRNP-enriched fraction (40P3). However, since U5 snRNA is resistant to micrococcal nuclease and RNase $H$ treatment (13), its requirement for complementation of inactivated prp18 extracts cannot be judged.

Isolation of heat-inactivated prp18 spliceosomes and chasing of the intermediates of splicing. It has been demonstrated by various means that the first step of splicing takes place only after the assembly of the spliceosome is complete $(8,17,23$, 31 ). It is of interest to know whether components required 


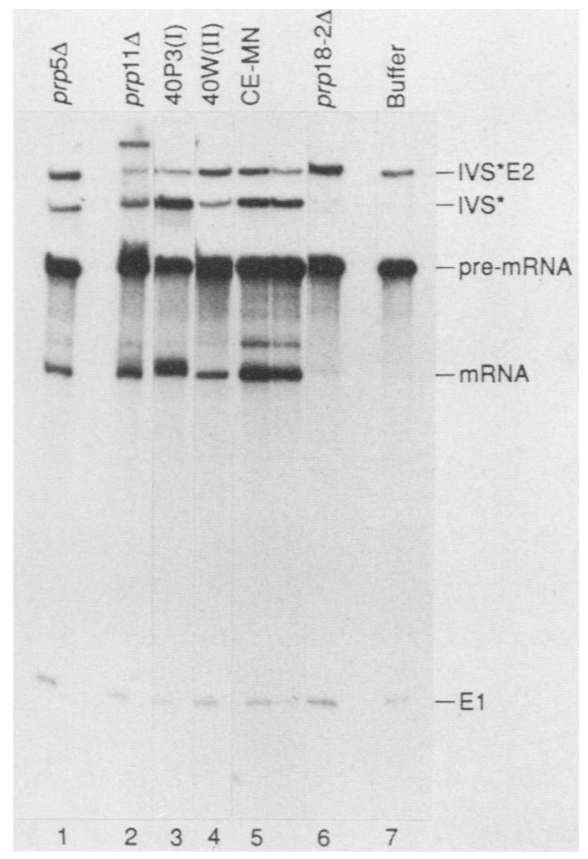

FIG. 2. Complementation of heat-inactivated prpl8 extract. prp18 ( $t s 503-6 \mathrm{c}$ ) extract, inactivated at $32^{\circ} \mathrm{C}$ for $70 \mathrm{~min}$, was assayed individually (lane 7) and in combination with either heat-inactivated $(\Delta)$ prp5 extract (lane 1) or prp11 (lane 2) or in combination with a different inactivated prpl8 (ts 503-1a) extract (lane 6). Combinations of prp 18 inactivated extracts and fractions of wild-type extract $40 \mathrm{P3}$ (lane 3) or 40W (lane 4) or micrococcal nuclease-treated wild-type extract (lane 5) were also assayed for complementation. After mixing of extracts, the splicing reactions were initiated with the addition of splicing cocktail and incubation at $15^{\circ} \mathrm{C}$ for $60 \mathrm{~min}$. The reaction intermediates and products are as described for Fig. 1A. Heat-inactivated extracts are indicated $(\Delta)$. In each case, when assayed individually, the inactivated mutant prp 5 or prpll extract or the complementing wild-type fraction was completely devoid of splicing activity (data not shown). The high-molecular-weight band seen in lane $\mathbf{2}$ is due to some nonspecific aggregation at the origin of the gel that was observed with some extracts.

for the second reaction can interact with preformed spliceosomes or whether they must interact early in spliceosome assembly. We have isolated spliceosomes formed in prp18 heat-inactivated extracts in order to investigate this question.

Large-scale splicing reactions performed with heat-inactivated prp 18 extract containing pre-mRNA, lariat intermediate, and exon 1 were sedimented through glycerol gradients, and fractions were collected and counted (Fig. 3A). The labeled pre-mRNA in the $40 \mathrm{~S}$ and the $30 \mathrm{~S}$ regions of the gradient was monitored by electrophoresis on a denaturing polyacrylamide gel. A peak of lariat intermediate, exon 1, and pre-mRNA was observed in the $40 \mathrm{~S}$ region of the gradient. This indicates that the defective spliceosomes remain reasonably intact and can be isolated by gradient sedimentation. Upon addition of inactivated prp5 or prpl1 extracts or the ammonium sulfate fraction $40 \mathrm{P} 3$ to the spliceosomal complexes in fraction 12 , a conversion to products was observed (Fig. 3B, lanes 1, 2, and 3). No products were formed in the absence of complementing extract (Fig. 3B, lane 7). The chasing of the spliceosomal intermediates is not complete, implying some inefficiency in complementation with assembled spliceosomes. Complementation of the inactivated spliceosome has a requirement for ATP (Fig. 3B, lane 8). This experiment demonstrates the requirement for ATP in the second step of the splicing reaction.

The complementation to chase intermediates into products could be due to (i) chasing of the lariat intermediate and exon 1 in the preformed complex, (ii) disassembly and reassembly of splicing components in the spliceosome and the complementing fraction, or (iii) the splicing of the pre-mRNA in this region of the gradient. In all cases in which chasing was observed, a decrease in the level of lariat intermediate accompanied the appearance of intron and mRNA. This supports our belief that chasing of the spliceosomal intermediates takes place. The second possibility has been ruled out by the addition of a second transcript during chasing reactions that, if spliced, would result in a differentsize mRNA. These chasing reactions did not result in any mRNA production from the second transcript (data not shown). We cannot rule out the possibility that pre-mRNAs in assembled spliceosomes were also chased into products.

As with the complementation, chasing of splicing intermediates is obtained with micrococcal nuclease-treated crude extracts or ammonium sulfate fraction 40P3 (Fig. 3B, lanes 4 and 5), again suggesting that neither complementation nor chasing requires intact snRNPs. These experiments demonstrate that PRP18 function is not needed for the formation of spliceosomes but is required for the second step of splicing. They further suggest that PRP18 can interact with the preassembled spliceosome.

Isolation of the PRP18 gene. Molecular analysis of the locus encoding the $P R P 18$ gene product is essential for the complete analysis of the role of this gene product in splicing. The PRPI8 gene was cloned on the basis of its ability to complement the temperature-sensitive mutation. A prpl8 strain was transformed with a yeast genomic library constructed in a yeast centromere plasmid library and incubated on selective plates at $23^{\circ} \mathrm{C}$. The resulting URA transformant colonies were replica plated to prewarmed rich plates (YPD) at $37^{\circ} \mathrm{C}$ to select temperature-resistant colonies. Yeast plasmids were recovered from several of these colonies and transferred to $E$. coli. The plasmids were propagated in $E$. coli, and preliminary restriction mapping indicated that the recombinant plasmids were related. Retransformation of the prp18 strain with these plasmids from the yeast library after propagation in $E$. coli confirmed that the presence of the clone confers temperature resistance. Transformation of the yeast vector YCp50 did not confer wild-type growth. One of the complementing plasmids (pUV18) was used in further experiments.

Strains bearing the plasmid pUV18 were patched on selective plates containing 5-fluoro-orotic acid to select for the loss of centromere-containing plasmids from the transformants. Ura colonies obtained by this method were tested for temperature sensitivity and were found to have reverted to the temperature-sensitive phenotype. Thus, the presence of the plasmid alone conferred a wild-type phenotype. Transformation of pUV18 into other prp strains did not result in complementation of the temperature-sensitive phenotype. All of these results indicate that pUV18 carries the PRP18 gene.

pUV18 contains a yeast genomic DNA fragment of about 7 kilobases (kb). A restriction map was generated. To define the limits of the complementing sequence, specific restriction fragments of the insert were subcloned into a yeast low-copy-number (CEN) shuttle plasmid (pPHY18). Figure 4 shows that while plasmids pUV18-2, pUV18-4, and pUV18-20 were capable of generating URA temperature- 


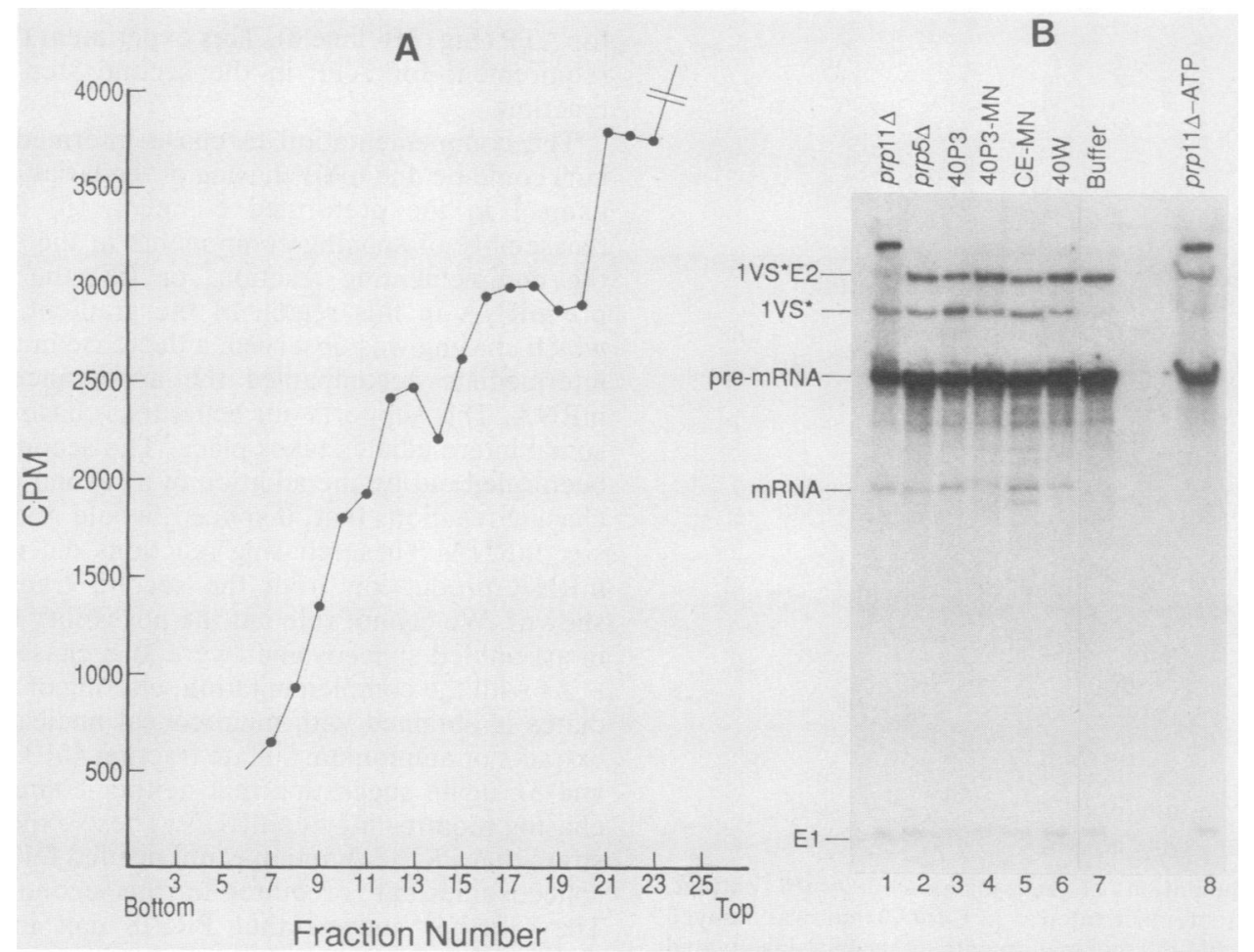

FIG. 3. (A) Chasing of heat-inactivated $(\Delta)$ prp18 spliceosomes. The gradient profile of a $50-\mu l$ splicing reaction conducted with inactivated prp18 extracts is shown. The reaction mixture was sedimented for $100 \mathrm{~min}$ and analyzed as described in Materials and Methods. Analysis of the labeled RNA in a sample of the gradient fractions (data not shown) revealed a peak of lariat intermediate at fraction 12, corresponding to the peak of ${ }^{32} \mathrm{P}$-labeled RNA in the $40 \mathrm{~S}$ region of the gradient. (B) Complementation of prpl $8(\Delta)$ spliceosomes. Fraction 12 from the gradient described in panel A was used as the prp18 heat-inactivated spliceosome. Chasing of the intermediates in the spliceosome was done as described in Materials and Methods. No complementing extract was added in lane 7, lane 1 was incubated with inactivated prp11, lane 2 was incubated with inactivated prp5, lane 3 was incubated with fraction 40P3 from wild-type extract, lane 4 was incubated with micrococcal nuclease-treated 40P3, lane 5 was incubated with $40 \mathrm{~W}$ fraction of wild-type extract, and lane 8 was incubated with inactivated prp11 extract in the absence of ATP in the chasing mix. The products of the chasing reaction, lariat intron (IVS*), and the spliced exons (mRNA) are indicated in the figure. Other products are as indicated for Fig. 1A.

resistant colonies and complementing the prp18 mutation when transformed into prp18, plasmids pUV18-3, pUV1817, and pUV18-19 yielded URA colonies that were temperature sensitive. These results imply that the complementing sequence lies in the BamHI-to-NruI region of the genomic fragment in pUV18. Subclone pUV18-20, which was capable of complementing the temperature-sensitive phenotype, was used in a second series of subcloning experiments, and several restriction fragments were cloned into the CEN vector pPHY18. These transformation experiments indicated that the HindIII site interrupts the complementing sequence but left unclear whether the $X h o I$ site lies within or outside the complementing sequence (Fig. 4). The complementing fragment (the region from BamHI to $\mathrm{NruI}$ ) was also cloned into a yeast multicopy vector $(2 \mu \mathrm{m}$, i.e., pSEY18) in addition to the low-copy-number vectors described above. The increased expression of this genomic fragment on this plasmid again gave complete complementation.

Complementation of the mutant splicing phenotype. The prp18 strain accumulates lariat intermediates of splicing in vivo at both permissive and nonpermissive temperatures (41). We have confirmed that this phenotype is relieved by the presence of the wild-type gene. Total RNA was prepared from the original prp18 strain, from the prp18 strains transformed with complementing plasmids (pUV18 and pUV1815 ), and from the strain transformed with the vector YCp50. The RNA was fractionated by electrophoresis on formaldehyde-agarose gels, blotted onto nylon membranes, and probed with a labeled fragment of the actin intron. Figure $5 \mathrm{~A}$, lanes 7 and 8 show accumulation of the lariat intermediate of actin in the original prpl8 strain and in the prpl8 strain bearing only the YCp50 vector (lanes 1 and 2) at both permissive and nonpermissive temperatures. No accumulation of lariat intermediate took place in the strain containing the original complementing clone pUV18 (Fig. 5, lanes 3 and 4) or the subclone pUV18-15 (lanes 5 and 6). In addition, reverse transcription experiments using a primer complementary to the RP51A intron 70 bases downstream of the $5^{\prime}$ splice site were done to detect lariat intermediates from the RP51A gene (for RP51A sequence, see reference 39). These experiments (Fig. 5B) again confirmed that the levels of lariat intermediate from the $R P 51 A$ gene are reduced to the wild-type levels (lane 1) in strains carrying pUV18 (Fig. 5B, lanes 2, 3, and 4). The mutant strain showed high levels of extension product corresponding to the lariat intermediate from $R P 51 A$ (Fig. 5B, lane 5).

Cloned DNA maps to the PRP18 locus. To prove that the complementing clone obtained corresponds to the $P R P 18$ gene and is not an extragenic suppressor capable of relieving the Prp phenotype, integration of the cloned complementing genomic DNA was performed. A complementing fragment (the 5-kb EcoRI fragment from pUV18-4) was cloned into a yeast integrating plasmid (YIP5). Directed integration of the YIP5 subcloned DNA was accomplished by restricting the DNA at the unique $X h o I$ site in the genomic fragment (Fig. 6A). URA colonies were selected after transformation of the 


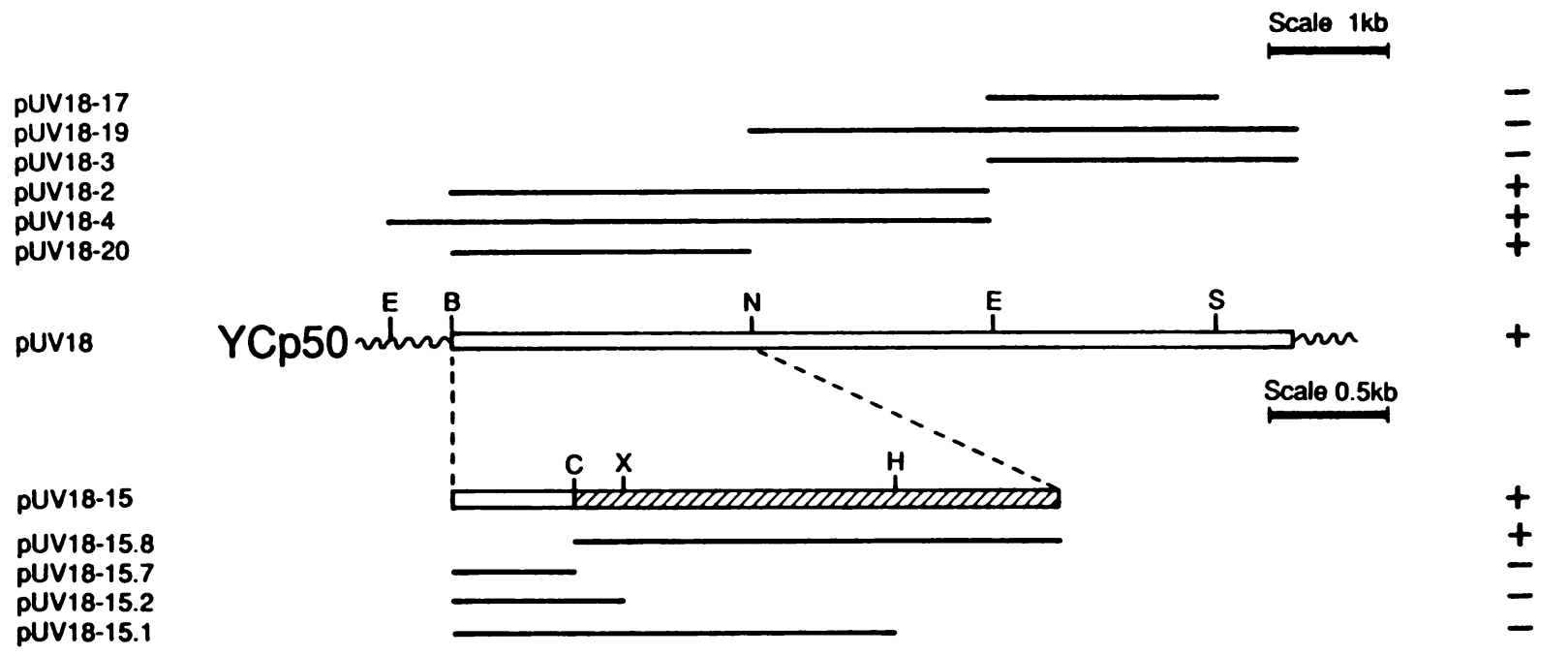

FIG. 4. In vivo complementation analysis with clones and subclones of PRP18. Restriction map of pUV18 and complementation phenotype for the subclones with portions of the genomic DNA fragment in pUV18 are shown. Only restriction sites relevant to the isolation of subclones and the generation of hybridization probes are indicated. Symbols: + , ability to suppress the temperature-sensitive growth defect of prp18; -, inability to confer temperature insensitivity; $m$, YCp50 sequences; and $\mathbb{E}$, complementing region. Restriction enzymes are indicated as follows: B, BamHI; C, ClaI; E, EcoRI; H, HindIII; N, NruI; S, SalI; and X, XhoI.

linear DNA into the wild-type SS330. The URA strains were crossed to the temperature-sensitive ura prpl8 strain, and diploids were selected for prototrophy. Sporulation of diploids and dissection of spores from two diploid clones demonstrated a cosegregation of the TS and URA phenotype and of the temperature-sensitive and Ura phenotype in several tetrads (14 from each diploid). This analysis shows that the cloned DNA was integrated at the homologous chromosomal PRPI8 locus.

Southern analysis was also performed as a second means of proving homologous recombination to the PRPI8 locus. Genomic DNA was isolated from the untransformed strains SS330 and prp18, two SS330 URA integrative transformants, and the diploid obtained from crossing the integrative transformant with prp18. All the genomic DNA preparations were digested with the enzyme SalI. The DNA was then separated by electrophoresis on agarose gels, blotted, and probed with a fragment containing the entire complementing region. Figure 6A diagrams the expected integration event to the $P R P 18$ locus. As expected, the probe detected only one SalI fragment (Fig. 6B) in the untransformed parental strains (the PRP18 gene does not contain a Sall site). Each integration event brings in one SalI site from the YIP5 vector sequences. Hence, as a result of integration the probe hybridized to two fragments in the haploid URA integrant SS330-Ib (Fig. 6B). Three fragments were detected in the SS330 integrative transformant SS330-Ia (Fig. 6B), an event most likely due to tandem insertion of two copies of the cloned DNA. All of the integration, segregation, and Southern blot analyses are consistent with our having cloned the wild-type allele of PRP18.

\section{DISCUSSION}

Identification of a factor affecting late stages of splicing. Biochemical fractionation of splicing extracts and analysis of the assembly of the spliceosomes have indicated the importance of snRNP and protein factors in splicing (reviewed in references 28 and 35 ). In the study of pre-mRNA splicing in yeast, a combined genetic and biochemical approach has been taken with the characterization of the PRP2 to PRPII gene products $(40,42)$. We have recently reported the generation of a bank of temperature-sensitive mutations, which were screened for defects in pre-mRNA splicing (41). One of the complementation groups with a novel splicing phenotype displayed an accumulation, in vivo, of the lariat intermediate. This phenotype resulted from a trans-acting defect affecting splicing and thus provided a means for the analysis of the components required specifically for the second step of splicing. In this paper we have investigated the role of the PRP18 gene product in vitro and have also obtained some molecular information about the PRPI8 gene.

Splicing extracts prepared from the mutant strain were defective in the second splicing reaction and accumulated the lariat intermediate and exon 1 . This splicing defect after heat inactivation of splicing extracts is the same as the in vivo phenotype after a shift to a nonpermissive temperature (41). The loss of activity for the $3^{\prime}$ intron-exon cleavage and exon ligation could result from specific inactivation of a splicing component or may be explained as a nonspecific effect. The second possibility has to be considered because some loss of splicing activity is observed upon heating of even wild-type extracts (26). However, this is unlikely for three reasons. First, loss of splicing activity in wild-type extracts, when observed, always diminishes total splicing activity due to a decrease in both reactions of splicing. Second, the in vitro loss of activity for the second step of splicing, observed in prp18 strains, can be complemented with inactivated extracts from different mutant strains and also with fractions of wild-type extracts. Restoration of activity in these mixing experiments probably results from the exchange of active components from each of the inacti- 


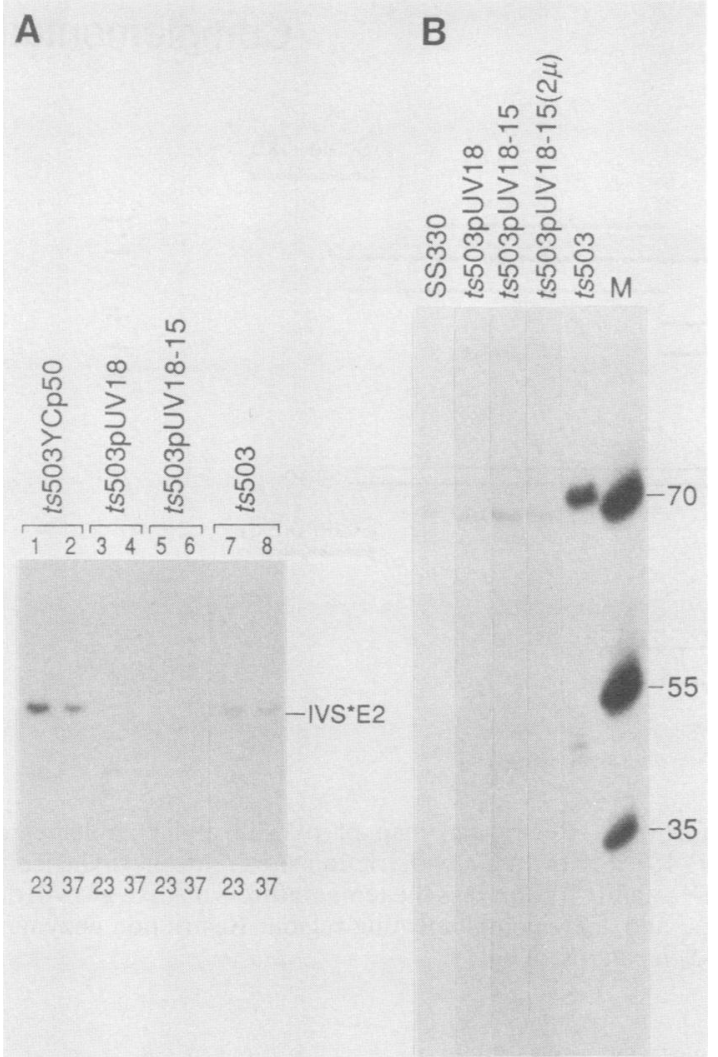

FIG. 5. (A) Complementation of lariat intermediate accumulation phenotype of prp18 strains. Total RNA (10 $\mu \mathrm{g}$ in each case) prepared from cultures at 23 or $37^{\circ} \mathrm{C}$ (indicated below the lanes) was electrophoresed through a $1.2 \%$ formaldehyde-agarose gel, blotted, and cross-linked to GeneScreen. The blot was then probed with a labeled fragment of the actin intron. Lanes 1 and 2 were loaded with RNA from a prp18 strain transformed with the vector YCp50, lanes 3 and 4 were loaded with RNA from prp18 transformed with the complementing subclone pUV18, lanes 5 and 6 were loaded with RNA from prp18 transformed with complementing subclone pUV18-15, and lanes 7 and 8 were loaded with RNA from the untransformed prp18 strain. The position of the actin lariat intermediate is indicated (IVS*E2). (B) Suppression of lariat intermediate phenotype of RP51A. Reverse transcription was done to map the 5' end of RP51A transcripts. Total RNA $(20 \mu \mathrm{g})$ was hybridized to an RP51A oligonucleotide complementary to sequences 70 nucleotides downstream from the $5^{\prime}$ splice site. The extension products were separated on $8 \mathrm{M}$ urea-10\% acrylamide gels. RNA from untransformed wild-type (lane 1) and prp18 (lane 5) strains, the prp18 strain transformed with the complementing plasmid pUV18 (lane 2), the prp18 strain transformed with the subclone pUV18-15 (lane 3), and the prp18 strain transformed with a complementing multicopy plasmid (lane 4) was used. The last lane (M) contains oligonucleotide markers of 70,55 , and 35 nucleotides treated with kinase, as indicated at right. The strong extension stop seen in $t s 503$ just above the 70-nucleotide marker corresponds to a reverse transcription stop at the $5^{\prime}$ end of the accumulated lariat intermediate in the mutant strain.

vated extracts. Third, the cloned PRPI8 gene completely suppresses the Prp18 phenotype. The results strongly imply that the splicing defect in prpl8 results from a specific inactivation of a splicing component.

Complementation of heat-inactivated prp18 extracts indicated that complementation could be achieved by the addition of wild-type fractions containing the PRP18 gene product. Fractionation of wild-type extracts by ammonium sulfate precipitation followed by heparin agarose chromatog-

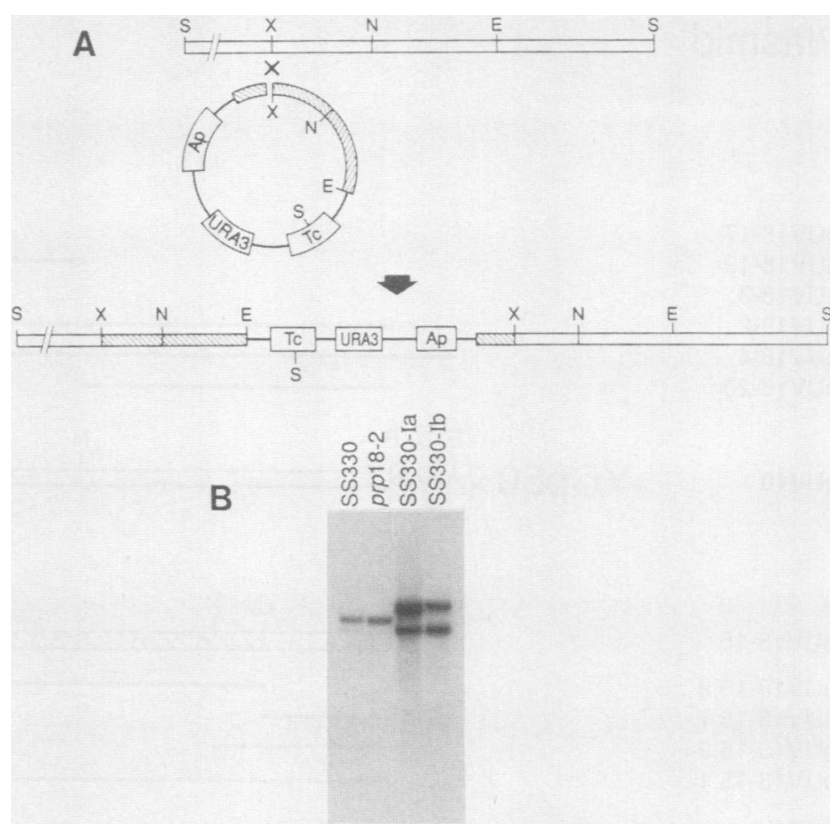

FIG. 6. Integration of the cloned DNA to the homologous chromosomal PRP18 locus. (A) Diagram of the expected integration event. A 5-kb complementing EcoRI fragment (Fig. 4) subcloned in YIP5 was linearized at the unique XhoI site and transformed in the wild-type strain SS330. Symbols: $\square$, chromosomal locus; cloned yeast DNA. Plasmid sequences (-) and markers in the plasmid (Ap, ampicillin resistance; Tc, tetracycline resistance; URA3, yeast marker gene) are indicated. Restriction sites are represented by the notations as given in the legend to Fig. 4. (B) Southern blot confirming integration event at the cloned genomic locus. DNA from the wild-type, prp 18 strain, and two integrants (SS330-Ia and -Ib) was digested with SalI and run on a $1.0 \%$ agarose gel, blotted, and probed with the 4.5-kb BamHI-to-EcoRI (Fig. 4) complementing fragment.

raphy has already identified three fractions (I, II, and III), necessary for splicing (7). Fractions I and II are together capable of assembling spliceosomes and supporting the first step of splicing. Fraction III is required for completion of splicing in the presence of fractions I and II. Complementation of heat-inactivated prpl8 extracts is best achieved with $40 \mathrm{P} 3$ (fraction I) and not with $40 \mathrm{~W}$ (fractions II and III). This indicated to us that there exists a requirement for components other than those present in fraction III for the completion of splicing in the heat-inactivated prp 18 extracts. The strong complementation obtained with $40 \mathrm{P} 3$ (fraction I) implied that the complementing component needed for prp 18 is enriched in wild-type fraction $I$.

PRP18 probably corresponds to one of the as yet unidentified but previously hypothesized factors, $c_{n}$. These factors, required for the second step of pre-mRNA splicing, were suggested from experiments in which prp2 heat-inactivated spliceosomes were complemented with wild-type extract fractions (23). In these studies chasing of heat-inactivated prp2 spliceosomes containing only pre-mRNA with $40 \mathrm{P} 3$ (fraction I) resulted in completion of both splicing reactions, while complementation of prp 2 spliceosomes with $40 \mathrm{~W}$ (fractions II and III) resulted in accumulation of lariat intermediate. These experiments indicated that some factor(s) other than those present in fraction III is required for the second step of splicing, and it was deduced to be enriched in 40P3 (fraction I). The complementation of heat- 
inactivated prp 18 extract by fraction I implies that PRP18 is possibly one of the $c_{n}$ factors.

Fractionation of HeLa cell extracts has identified splicing factors required for the second step of splicing (19). One component (SF3) identified by complementation assays was described as a heat-sensitive factor present in nuclear extracts. In a preliminary fractionation scheme, SF3 cofractionated with snRNP-containing fractions. The other component required for the second step of splicing (SF4A) was identified in fractions depleted in snRNPs. The role of SF3 and SF4A in splicing reactions is similar to that of PRP18. Both SF3 and PRP18 are enriched in snRNP-enriched fractions. These two factors may play an analogous role. To know whether they are homologous will require further work in both systems. Although PRP18 appears to be enriched in an snRNA-enriched fraction, micrococcal nuclease-treated wild-type extract or 40P3 (fraction I) retains complementing activity. This suggests the dispensibility of intact $U 1, U 2$, U4, and U6 spliceosomal snRNAs. These results do not rule out the possibility that PRP18 is a protein component of one of these snRNP(s) or a dissociable part of a multicomponent structure. However, PRP18 must be exchangeable with the mutant protein even after assembly of the spliceosome. The RNase $\mathrm{H}$ and micrococcal nuclease insensitivity of the essential, spliceosomal snRNA U5 make it difficult to establish whether it must remain intact for complementation of inactivated prpl8 extracts. A direct assessment of any association of PRP18 with snRNAs will be possible with antibodies raised against the PRP18 protein.

prp18 spliceosomes an intermediate in the splicing pathway. The in vitro complementation of heat-inactivated prp18 extracts implies that splicing components can exchange. The formation of the spliceosome takes place even after heat inactivation, suggesting that the PRP18 function is dispensable for spliceosome formation. Experiments were designed to test whether the lariat intermediate in the spliceosome could be chased to products and thus to determine whether these spliceosomes represent intermediates in splicing. Chasing of the intermediates of splicing into product is achieved, although inefficiently, in the presence of complementing factors. A requirement for ATP was observed in the complementation reaction.

Multiple roles for ATP in splicing have been observed for the assembly of the spliceosome and for the first reaction of splicing $(8,17,23,31)$. The requirement for ATP in the final step of splicing, $3^{\prime}$ cleavage, and exon ligation had not been previously shown. We have demonstrated that ATP and a complementing extract are required in order to chase the intermediates in a prp18 spliceosome into products. Since the gradient fractions that contain the intermediates most probably also have spliceosomes which contain pre-mRNA, we cannot exclude the possibility that this assembled premRNA is also being chased into products. The correlation between the appearance of mRNA and the decrease in the lariat intermediate supports our belief that chasing of the intermediates into products occurs. Recent experiments with $\mathrm{HeLa}$ extracts have reported a requirement for ATP for the second reaction of splicing (33). In these experiments, the second splicing reaction was selectively inhibited, and completion of splicing in such extracts occurred only in the presence of ATP and additional nuclear extract. As in our experiments, the isolated complex containing intermediates could only form mRNA if ATP as well as extract was added. These results taken together strongly suggest that ATP is required for the second splicing step in both yeast and HeLa systems. It will be very interesting to know what the biochemical role of ATP is in this step and in any of the several steps in which it is required.

Molecular analysis of PRP18. The temperature sensitivity of the prp18 mutation has allowed the cloning of the wildtype gene, and this was essential for further analysis of the role of PRP18 in splicing. The cloned gene in single-copy or low-copy-number vectors (i.e., CEN vectors [YCp50 and pPHY18]) is capable of rescuing the temperature-sensitive phenotype and the in vitro splicing defect. The cloned DNA has been characterized by restriction mapping and subcloning; $\mathrm{a} \sim 2.0-\mathrm{kb}$ genomic fragment has been shown to complement both phenotypes. This fragment is unique to the yeast haploid genome. The cloned DNA has been shown to be genetically linked to the PRP18 locus by integrative transformation followed by segregation and Southern analysis. These studies showed that the cloned DNA is the wild-type $P R P 18$ gene and not a suppressor of the temperature-sensitive and splicing phenotype. Transformation of the cloned DNA on multicopy vectors does not alter the complementation of phenotypes, and hence overexpression of the protein-using yeast vectors would probably not be deleterious to the cell.

Further experiments to characterize this new yeast splicing factor, including sequencing of the PPR 18 gene, characterization of the transcript, and analysis of the gene product, are in progress.

\section{ACKNOWLEDGMENTS}

We thank D. Horowitz and J. Banroques for their critical reading and comments on the manuscript.

This work was supported by Public Health Service grant GM 32637 from the National Institutes of Health.

\section{LITERATURE CITED}

1. Banroques, J., and J. Abelson. 1989. PRP4: a protein of the yeast U4/U6 small nuclear ribonucleoprotein particle. Mol. Cell. Biol. 9:3710-3719.

2. Bindereif, A., and M. R. Green. 1986. Ribonucleoprotein complex formation during pre-mRNA splicing in vitro. Mol. Cell. Biol. 6:2582-2592.

3. Bindereif, A., and M. R. Green. 1987. An ordered pathway of snRNP binding during mammalian pre-mRNA splicing complex assembly. EMBO J. 6:2415-2424.

4. Boeke, J. D., F. LaCroute, and G. Fink. 1984. A positive selection for mutants lacking orotidine-5'-phosphate decarboxylase activity in yeast: 5-fluoro-orotic acid resistance. Mol. Gen. Genet. 197:345-346.

5. Brody, E., and J. Abelson. 1985. The "spliceosome": yeast pre-mRNA associates with a $40 \mathrm{~S}$ complex in a splicing dependent reaction. Science 228:963-967.

6. Chang, T.-H., M. W. Clark, A. J. Lustig, M. E. Cusick, and J. Abelson. 1988. RNA11 protein is associated with yeast spliceosome and is localized in the periphery of the cell nucleus. Mol. Cell. Biol. 8:2379-2393.

7. Cheng, S.-C., and J. Abelson. 1986. Fractionation and characterization of yeast mRNA splicing extract. Proc. Natl. Acad. Sci. USA 83:2387-2391.

8. Cheng, S.-C., and J. Abelson. 1987. Spliceosome assembly in yeast. Genes Dev. 1:1014-1027.

9. Feinberg, A. P., and B. Vogelstein. 1983. A technique for radiolabeling DNA restriction endonuclease fragments to high specific activity. Anal. Biochem. 132:6-13.

10. Frendewey, D., and W. Keller. 1985. Stepwise assembly of pre-mRNA splicing complex requires U-snRNPs and specific intron sequences. Cell 42:355-367.

11. Grabowski, P. J., S. R. Seiler, and P. A. Sharp. 1985. A multicomponent complex is involved in splicing of messenger RNA precursors. Cell 42:345-353.

12. Green, M. R. 1986. Pre-mRNA splicing. Annu. Rev. Genet. 
20:671-708.

13. Guthrie, C., and B. Patterson. 1988. Spliceosomal snRNAs. Annu. Rev. Genet. 22:387-418.

14. Ito, H., Y. Fukada, K. Murata, and A. Kimura. 1983. Transformation of intact yeast cells treated with alkali cations. J. Bacteriol. 153:163-168.

15. Jackson, S. P., M. Lossky, and J. D. Beggs. 1988. Cloning of RNA8 of Saccharomyces cerevisiae, detection of the RNA8 protein, and demonstration that it is essential for nuclear pre-mRNA splicing. Mol. Cell. Biol. 8:1067-1075.

16. Konarska, M. M., and P. A. Sharp. 1986. Electrophoretic separation of complexes involved in the splicing of precursors to mRNAs. Cell 46:845-855.

17. Konarska, M. M., and P. A. Sharp. 1987. Interactions between small nuclear ribonuclear particles in the formation of spliceosomes. Cell 49:763-774.

18. Kraemer, A., M. Frick, and W. Keller. 1987. Separation of multiple components of HeLa cell nuclear extracts required for pre-mRNA splicing. J. Biol. Chem. 262:17630-17640.

19. Krainer, A. R., and T. Maniatis. 1985. Multiple factors including small nuclear ribonucleoproteins U1 and U2 are necessary for pre-mRNA splicing. Cell 42:725-736.

20. Last, R. L., J. B. Stavenhagen, and J. L. Woolford. 1984. Isolation and characterization of the RNA2, RNA3, and RNAII genes of Saccharomyces cerevisiae. Mol. Cell. Biol. 4:23962405 .

21. Last, R. L., and J. L. Woolford. 1986. Identification and nuclear localization of yeast pre-mRNA processing components: $R N A 2$ and $R N A 3$ proteins. J. Cell Biol. 103:2103-2112.

22. Lee, M. G., R. A. Young, and J. D. Beggs. 1984. Cloning of the RNA2 gene of Saccharomyces cerevisiae. EMBO J. 3:28252830.

23. Lin, R.-J., A. J. Lustig, and J. Abelson. 1987. Splicing of yeast nuclear pre-mRNA in vitro requires functional $40 \mathrm{~S}$ spliceosomes and several extrinsic factors. Genes Dev. 1:7-18.

24. Lin, R.-J., A. J. Newman, S.-C. Cheng, and J. Abelson. 1985. Yeast mRNA splicing in vitro. J. Biol. Chem. 260:14780-14792.

25. Lossky, M., G. J. Anderson, S. P. Jackson, and J. D. Beggs. 1987. Identification of yeast snRNP protein and the detection of snRNP-snRNP interactions. Cell 51:1019-1026.

26. Lustig, A. J., R.-J. Lin, and J. Abelson. 1986. The yeast RNA gene products are essential for mRNA splicing in vitro. Cell 47:953-963.

27. Maniatis, T., E. F. Fritsch, and J. Sambrook. 1982. Molecular cloning: a laboratory manual. Cold Spring Harbor Laboratory, Cold Spring Harbor, N.Y.
28. Maniatis, T., and R. Reed. 1987. The role of small nuclear ribonucleoproteins in pre-mRNA splicing. Nature (London) 325:673-678.

29. Padgett, R. A., P. J. Grabowski, M. M. Konarska, S. Seiler, and P. A. Sharp. 1986. Splicing of messenger RNA precursors. Annu. Rev. Biochem. 55:1119-1150.

30. Perkins, K. K., H. M. Furneaux, and J. Hurwitz. 1986. RNA splicing products formed with isolated fractions from HeLa cells are associated with fast sedimentation complexes. Proc. Natl. Acad. Sci. USA 83:887-891.

31. Pikielny, C. W., B. C. Rymond, and M. Rosbash. 1986. Electrophoresis of ribonucleoproteins reveals an ordered assembly of yeast splicing complexes. Nature (London) 324:341-345.

32. Ruby, S. R., and J. Abelson. 1988. An early and hierarchic role for U1 snRNP in spliceosome assembly. Science 24:1028-1035.

33. Sawa, H., M. Ohno, H. Sakamoto, and Y. Shimura. 1988. Requirement for ATP in the second step of the pre-mRNA splicing reaction. Nucleic Acids Res. 16:3157-3164

34. Shapiro, M. B., and P. Senapathy. 1987. RNA splice junctions of different classes of eukaroyotes: sequence statistics and functional implications in gene expression. Nucleic Acids Res. 15:7155-7174.

35. Sharp, P. A. 1987. Splicing of messenger RNA precursors. Science 235:766-771.

36. Sherman, F., G. Fink, and J. Hicks. 1986. Methods in yeast genetics. Cold Spring Harbor Laboratory, Cold Spring Harbor, N.Y.

37. Soltyk, A., M. Tropak, and J. D. Friesen. 1984. Isolation and characterization of the RNA2, RNA4, and RNA11 genes of Saccharomyces cerevisiae. J. Bacteriol. 160:1093-1100.

38. Steitz, J. A., D. L. Black, V. Gerke, K. A. Parker, A. Kraemer, D. Frendewey, and W. Keller. 1987. Function of the abundant U-snRNPs, p. 115-154. In M. Birnsteil (ed.), Structure and function of major and minor SNURPS. Springer-Verlag, New York.

39. Teem, J. L., and M. Rosbash. 1983. Expression of $\beta$-galactosidase gene containing ribosomal protein 51 intron is sensitive to the rna2 mutation of yeast. Proc. Natl. Acad. Sci. USA 80: 4403-4407.

40. Vijayraghavan, U., and J. Abelson. 1989. Pre-mRNA splicing in yeast. Nucleic Acids Mol. Biol. 3:197-215.

41. Vijayraghavan, U., M. Company, and J. Abelson. 1989. Isolation and characterization of pre-mRNA splicing mutants in Saccharomyces cerevisiae. Genes Dev. 3:1206-1216.

42. Warner, J. R. 1987. Applying genetics to the splicing problem. Genes Dev. 1:1-3. 